\title{
La remédiation des faibles compétences de raisonnement clinique ${ }^{\star}$
}

\section{The remediation of weak clinical reasoning skills}

\author{
Jeannette GUERRASIO* et Juan N. LESSING \\ University of Colorado School of Medicine, Aurora, Colorado, USA
}

Manuscrit soumis le 31 octobre 2017 ; commentaires éditoriaux formulés aux auteurs le 24 octobre 2017 ; accepté pour publication le 31 octobre 2017

\begin{abstract}
Résumé-Problématique : Le débat se poursuit quant à savoir si le raisonnement clinique peut être enseigné et appris ou si le raisonnement clinique est une compétence ou un trait constitutionnel. Un programme de remédiation du raisonnement clinique a permis d'observer qu'un tiers de tous les stagiaires étudiants et résidents référés pour un enseignement de remédiation avaient de faibles capacités de raisonnement clinique, bien qu'ils possédaient des connaissances suffisantes pour réussir les cours et les examens de licence. Les étudiants peu performants font preuve de plusieurs faiblesses générales, liées notamment à des apprentissages purement opportunistes ou à l'absence de cadre adéquat permettent de structurer et d'organiser leurs connaissances; ils éprouvent aussi des difficultés à apprendre dans le cadre du curriculum caché ou à identifier les commentaires non explicites. Exégèse: Dans cet article, nous décrivons un processus étape par étape pour identifier, diagnostiquer et corriger des faiblesses en termes de capacité de raisonnement clinique. Bien que l'utilisation de cette méthode de remédiation spécifique ait montré sa capacité à améliorer les aptitudes des étudiants en difficulté, des développements ultérieurs seront nécessaire pour vérifier si elle est en mesure de conduire ces étudiants à un niveau d'excellence en matière de raisonnement clinique.
\end{abstract}

Mots clés : raisonnement clinique, remédiation, compétence

\begin{abstract}
Context: Debate continues as to whether clinical reasoning can be taught and learned or if clinical reasoning is an innate skill or trait. One remediation program noted that one-third of all student and resident trainees referred for remedial teaching had weak clinical reasoning skills despite possessing adequate knowledge to pass courses and licensing exams. Underperforming students display several universal weaknesses, including: haphazard learning and absence of a framework on which to structure and organize their knowledge; difficulty learning from the hidden curriculum; and difficulty identifying non-explicit feedback. Analysis: In this article, we describe a step-by-step process to identify, diagnose, and remediate weak clinical reasoning skills. While use of this specific remediation method on struggling learners did confer the necessary skills for competent practice, further development in methods is needed to see if struggling learners can go on to excel in the domain of clinical reasoning.
\end{abstract}

Keywords: clinical reasoning, remediation, competency

\section{Introduction}

Lors de leur formation en sciences de la santé, les étudiants ou les internes commencent par apprendre une

\footnotetext{
‡ Ce texte a été élaboré à partir d'une conférence plénière prononcée dans le cadre de la troisième Conférence internationale de Montréal sur le raisonnement clinique qui s'est tenue à Montréal du 25 au 28 octobre 2016. La traduction en français a été effectuée par Marie-Claude Audétat en collaboration avec Annie Carrier, Joseph-Omer Dyer, Caroline Faucher, Stuart Lubarsky et Meredith Young et en lien avec le comité de rédaction de la revue. Correspondance et offprints: Jeannette GUERRASIO

*Mailto: Jeannette.Guerrasio@ucdenver. edu
}

série de faits et de concepts liés aux différents systèmes et organes. Ils sont ensuite évalués au moyen d'une série de tests, élaborés, pour la plupart, afin de vérifier qu'ils maîtrisent bien les connaissances indispensables à leur pratique. Les apprenants essaient ensuite d'appliquer leurs connaissances médicales à des situations cliniques. Ainsi, si un patient se présente par exemple avec un «nez qui coule », l'étudiant va réfléchir aux causes possibles de cette rhinorrhée (par exemple des allergies ou des infections telles qu'un banal rhume ou une sinusite). Puis il tentera d'expliquer les symptômes du patient en les mettant en lien avec ses connaissances et en les catégorisant au regard de ce qu'il sait du diagnostic différentiel associé à ces symptômes. 
Néanmoins, posséder des connaissances médicales ne confère pas nécessairement la capacité d'appliquer adéquatement ces connaissances à une situation clinique spécifique qui concerne un patient unique. Ainsi, si un étudiant peut être tout à fait capable de fournir des détails très précis sur la physiopathologie des allergies et du rhume, il peut en revanche devenir quasiment «paralysé » quand un patient lui décrit un symptôme plutôt que le diagnostic.

La plupart des apprenants des professions de la santé arrivent à développer adéquatement les compétences cruciales de raisonnement clinique, même s'ils reçoivent peu de consignes explicites sur la façon dont ils devraient procéder pour «raisonner» à partir des cas cliniques qui se présentent à eux. Cependant, une minorité de ces apprenants rencontre des difficultés à développer cette compétence. Une de nos recherches sur un programme de remédiation a mis en évidence qu'un tiers de tous les étudiants et internes référés au programme de remédiation éprouvaient des difficultés de raisonnement clinique et ceci, bien qu'ils disposaient des connaissances suffisantes pour réussir leurs cours et leurs examens de certification [1].

Il n'y a pas de consensus quant au fait de savoir si le raisonnement clinique peut être enseigné et appris, ou s'il s'agit au contraire d'une aptitude constitutionnelle [2]. Considérant la nécessité d'aider les étudiants et internes en difficulté, l'auteur de cette communication (JG), en collaboration avec Eva et Aagaard, a développé et implanté une stratégie par étapes pour l'enseignement du processus de raisonnement clinique auprès d'étudiants sous-performants, intitulée «Méthodes pour la remédiation du raisonnement clinique», qui avait pour objectif de vérifier la possibilité que le raisonnement clinique puisse être enseigné et appris.

\section{Objectifs}

Cette communication a pour intention de partager avec les lecteurs nos constats quant aux caractéristiques des apprenants sous-performants qui se dégagent de nos récents travaux. Par ailleurs, nous décrirons les disparités potentielles entre la façon dont nous enseignons et la façon dont les étudiants sous-performants apprennent. Enfin, nous présenterons les résultats nuancés obtenus dans des projets en cours qui visent à enseigner le raisonnement clinique.

\section{Résultats / Discussion \\ Comment fonctionnent nos apprenants sous- performants?}

Les étudiants sous-performants présentent plusieurs faiblesses générales communes

Premièrement, leur apprentissage est désorganisé et manque d'une assise solide, c'est-à-dire d'une structure dans laquelle ils peuvent organiser leurs connaissances. Ainsi, ces étudiants ont tendance à mémoriser des faits et des concepts mais ils ne perçoivent pas comment ces informations sont reliées entre elles. Par exemple, ils ne vont pas identifier par eux-mêmes en quoi la rhinorrhée allergique peut être reliée à la sinusite ou comment une maladie virale pourrait se présenter comme une sinusite, ou potentiellement conduire à une sinusite. En fait, ils envisagent chaque diagnostic potentiel comme étant isolé, unique, défini et indépendant de tout autre diagnostic.

Deuxièmement, ces étudiants n'apprennent pas à partir du curriculum caché. Le «curriculum caché/hidden curriculum» désigne les apprentissages faits de manière informelle au travers de tout «ce qui est effectivement fait sans toutefois être explicité» au sein d'un curriculum de formation. Les étudiants font preuve d'un apprentissage inconscient limité et d'un faible esprit de synthèse. Le fait par exemple, d'observer un superviseur conduisant une entrevue clinique avec un patient, et aboutissant à un diagnostic de rhinite allergique, ne va pas leur permettre de se faire une idée précise du diagnostic différentiel sousjacent, ni de raisonner adéquatement et de prioriser les bonnes hypothèses cliniques dans un cas analogue par la suite.

Enfin, ces étudiants peinent à identifier le fait qu'on leur donne une rétroaction, à moins que ce ne leur soit explicitement spécifié. Et même lorsque cette rétroaction est explicite, ils ont du mal à intégrer et transférer ces commentaires, ce qui entrave, entre autres choses, le développement de leurs compétences de raisonnement clinique.

\section{Inadéquation entre l'enseignement et l'apprentissage}

Nous pouvons distinguer cinq écarts majeurs entre notre façon d'enseigner et celle avec laquelle nos étudiants en difficulté apprennent:

- nous enseignons souvent en nous centrant davantage sur le contenu didactique que sur le niveau de compétences de l'apprenant. Par exemple, nous commençons notre enseignement en décrivant les éléments clés de l'examen et du diagnostic d'un patient atteint de rhinorrhée et ce, avant même d'évaluer ce que sait ou comprend l'étudiant à propos de la rhinorrhée, ou s'il est compétent pour procéder à un examen clinique adéquat;

- lorsque nous enseignons à partir de la situation clinique d'un patient souffrant de rhinite allergique, par exemple, nous prenons pour acquis que l'étudiant possède déjà une organisation des connaissances dans laquelle il pourra intégrer ces connaissances nouvellement acquises. Or, ce n'est pas toujours le cas pour les étudiants en difficulté chez qui la désorganisation des connaissances de base peut nuire à l'acquisition de nouvelles connaissances;

- nous nous attendons à ce que nos apprenants déduisent par eux-mêmes des comportements appropriés, de même que notre raisonnement, et ce simplement en nous observant. Or, les apprenants en difficulté ont besoin que nous leur disions explicitement et démontrions 
concrètement ce que nous attendons d'eux. Par exemple, à moins qu'il ne leur soit explicitement dit d'élaborer leur diagnostic différentiel en se basant prioritairement sur les informations fournies par le patient, telles que son âge, son genre, sa plainte principale, les étudiants en difficulté vont avoir tendance à entamer une démarche systématique et peu adaptée de collecte exhaustive d'informations. Ainsi, ces apprenants ne tentent de faire une synthèse de ces informations qu'à la toute fin de la collecte;

- les superviseurs se donnent généralement beaucoup de peine pour observer leurs apprenants et leur donner une rétroaction appropriée. Cependant, les étudiants en difficulté n'identifient pas nécessairement que le superviseur est en train de leur donner de la rétroaction pour qu'ils s'améliorent. Ainsi, ils ne vont pas être capables d'apprendre à partir de cette interaction avec les superviseurs, à moins que des consignes très claires concernant des actions spécifiques ne leur soient données sous la forme d'une rétroaction. De fait, plus les consignes seront claires et précises, meilleur sera le bénéfice pour l'apprenant. Un superviseur aura par exemple avantage à dire: «Voici ma rétroaction: Tu n'as pas examiné les oreilles de ce patient qui souffre de rhinorrhée. Chaque fois que tu vois un patient qui souffre de rhinorrhée, tu dois te faire une idée des membranes du tympan»;

- les superviseurs savent l'importance d'instaurer un environnement pédagogique de qualité. Ils sont convaincus d'y parvenir mais, en dépit de leurs efforts, les étudiants en difficulté craignent de se sentir ridicules et, de ce fait, sont peu enclins à discuter ouvertement de leurs processus de raisonnement clinique. Cette attitude restreint leurs possibilités, non seulement de recevoir la nécessaire confirmation ou redirection de leurs actions, mais aussi d'enrichir progressivement leurs scripts cliniques et de développer ainsi leurs compétences.

Lors de la conception d'un processus de remédiation «étapes par étapes», nous avons été très attentifs à réduire autant que possible les écarts entre l'enseignement et l'apprentissage décrits plus haut [3] (Tableau I). Notre processus démarre dès les premiers stades du raisonnement clinique, en ne présumant ainsi d'aucune compétence déjà établie dans ce domaine. Si les étudiants font preuve de compétences déjà acquises, ils peuvent alors se rendre plus rapidement aux étapes suivantes. Différents cadres de référence pour organiser leurs connaissances sont proposés aux apprenants, à partir de la plainte principale du patient et de leurs préférences personnelles. Toutes les consignes et tous les enseignements sont donnés de façon concrète, en procédant par étapes et en utilisant des tableaux et des algorithmes. Des méthodes pour améliorer la rétroaction directe sont utilisées à chaque étape du processus. Les enseignements se font de façon individuelle par un superviseur qui ne sera pas impliqué dans le cursus académique des apprenants par la suite
Tableau I. Écarts relevés entre la manière dont l'enseignement est dispensé et les caractéristiques de l'apprentissage des étudiants en difficulté.

\begin{tabular}{ll}
\hline Enseignement & Apprentissage \\
\hline Tâche d'enseignement & Compétence de l'apprenant \\
Assomption d'un cadre & $\begin{array}{l}\text { Absence de cadre } \\
\text { d'organisation des }\end{array}$ \\
connaissances & connaissances \\
Apprentissage inconscient/ & Nécessite un apprentissage \\
abstrait & concret \\
Rétroaction fournie & Rétroaction non-perçue \\
Environnement & Peur du ridicule \\
d'apprentissage sécurisant & \\
\hline
\end{tabular}

Une fois que la méthode de remédiation a été mise en œuvre de façon complète, les apprenants sont réévalués par des enseignants ou par une équipe d'enseignants qui ne sont pas au courant du processus de remédiation suivi par l'apprenant. Cette évaluation comprend différents temps et méthodes d'évaluation, tels que l'observation de rencontres cliniques avec des patients standardisés, l'administration de tests de concordance de scripts [4], la répétition de tout ou d'une partie d'un stage clinique et des évaluations avec les Mini-CEX (Mini clinical evaluation exercise); il s'agit en l'occurrence d'évaluations à partir d'une observation directe de l'interaction étudiantpatient. De plus, la méthode comprend des moments de discussion et d'explicitation de leur raisonnement clinique à partir de dossiers cliniques et, enfin, l'évaluation de leurs stages par les superviseurs habituels. Quatre-vingt-seize pourcent des apprenants ayant suivi le processus de remédiation ont réussi cette évaluation de leurs compétences qui a été effectuée de manière indépendante du programme de remédiation. Il est intéressant de noter que, si les étudiants en difficulté se sont avérés à même de développer leurs compétences en raisonnement clinique, leurs progrès n'ont cependant pas dépassé la moyenne des résultats de leur groupe de pairs.

Il faut relever néanmoins que ces apprenants sont devenus en mesure d'identifier leurs limites et d'oser demander plus facilement et plus fréquemment du soutien auprès de leurs pairs et des médecins spécialistes dans leur environnement de formation.

\section{Conclusion et message-clés}

Davantage d'attention doit être accordée non seulement aux difficultés des apprenants sous-performants, mais aussi à leurs besoins spécifiques d'apprentissage, de façon à les aider à surmonter leurs difficultés de raisonnement clinique. La méthode de remédiation que nous avons utilisée a permis aux étudiants concernés de développer les compétences nécessaires à leur pratique. Néanmoins, de futurs développements de cette méthode permettront d'investiguer s'il est possible d'amener les étudiants en difficulté à s'améliorer au-delà des perfor- 
mances moyennes de leur groupe académique, voire de faire preuve d'excellence dans leurs processus de raisonnement clinique.

\section{Références}

1. Guerrasio J, Garrity MJ, Aagaard EM. Learner deficits and academic outcomes of medical students, residents, fellows and physicians referred to a remediation program, 2006-2012. Acad Med 2014;89:352-8.

2. Schuwirth L. Can clinical reasoning be taught or can it only be learned? Med Educ 2002;36:695-6.

3. Guerrasio J, Aagaard EM. Methods and outcomes for the remediation of clinical reasoning. J Gen Intern Med 2014;29:1607-14.

4. Lubarsky S, Dory V, Duggan P, Gagnon R, Charlin B. Script concordance testing: from theory to practice: AMEE Guide No. 75. Med Teach 2013;35:184-93.

Citation de l'article : Guerrasio J., Lessing J.N., La remédiation des faibles compétences de raisonnement clinique. Pédagogie Médicale 2017:18;3-6 